\title{
Quadrant Based WSN Routing Technique by Shifting of Origin
}

\author{
Nandan Banerji, M.Tech(CSE) \\ Department of Computer science \& Engineering, \\ University of Kalyani, Nadia, W.B, India
}

\author{
Pulak Majumder(Astt professor), \\ Department of Electronics and Tele-Communication \\ Engineering, Regent Education \& Research Foundation, \\ Barrack pore, W.B, India
}

Abstract-A sensor is a miniaturized, low powered (basically natural phenomenon or things and convert it into electrical energy or vice versa using transduction process. A Wireless Sensor Network (WSN) is such a wireless network built using sensors. The sensors communicate with each other's using wireless medium. They can be deployed in such an environment; inaccessible to human or difficult to reach. Basically there is a vast application on automated world such as robotics, avionics, oceanographic study, space, satellites etc. The routing of a packet from a source node to a destination should be efficient in such a way that must be efficient in case of energy, communication overhead, less intermediate hops. The scheme will help to route the packet with a lesser intermediate nodes as the neighbors are being selected based on their Quadrant position.

\section{Keywords-component; WSN; Quardent; Packet; Hops}

\section{INTRODUCTION}

WSN is a special case of Mobile Ad-hoc NETwork (MANET) where the network topology changes very frequently with a very limited battery power. So routing in such a network is very difficult due to the dynamic network configuration. The nodes are scattered in such a manner and they are moving around the network that it is very difficult to predict the physical position of a node at a particular time.One of the related issue is that different nodes have different mobility

patterns; i.e. some nodes are heavily mobile, while some others are not. It is very difficult to predict the node movement within a WSN.

The basic characteristics of a WSN are :

1) Topology that changes dynamically.

2) Variable link capability and constraint of bandwidth of the wireless medium.

3) Optimization criteria of battery power.

4) Physical security limitations.

In our paper we have proposed an idea of routing in a WSN. In section-I there is a brief introduction, Section-II Related work in this domain, Section-III Idea of the proposed

\author{
Uttam Kumar Kundu (Principal) \\ Bengal College of polytechnic, Bidhan Nagar \\ Durgapur, W.B, India \\ Debabrata Sarddar (Asst. Professor) \\ Department of Computer science \& Engineering, \\ University of Kalyani, Nadia, W.B, India
}

work \& in Section-IV, V and VI the simulation, Acknowledgement, future work are briefly mentioned.

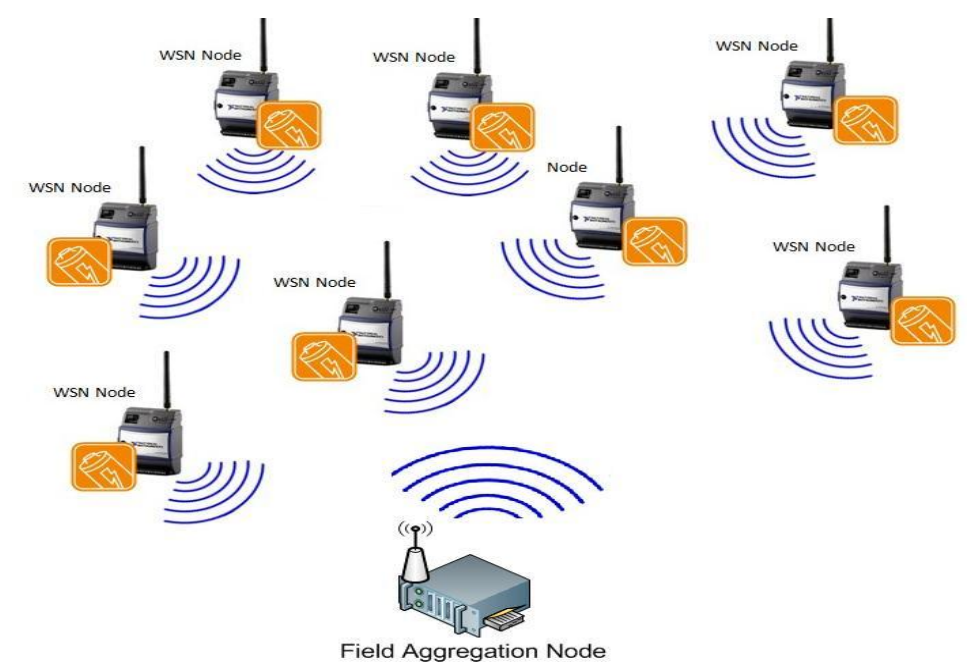

Fig.1. WSN

\section{RELATED WORK:}

The existing routing protocols are either proactive or reactive in nature .In proactive scheme the route is evaluated continuously hence at the time of packet forwarding time the route is known. On the other hand in reactive scheme the route is evaluated when it is demanded (sometimes it is called on demand routing). The protocols are also classified as:

\section{$>$ On Demand or Source initiated. \\ $>$ Table Driven.}

The On demand includes AODV, DSR, TORA, ASR and Table driven includes CGSR, DSDV, and WRP.

Most of the routing techniques in WSN are categorized as Geographic Routing, Hierarchical Routing, Stateless Flat Routing and Energy Aware Routing. The available schemes are of Hierarchical routing which dealing with clusters and $\mathrm{CH}$ (Cluster Heads). These schemes divide the network into 
regions/segments and then route the traffic from one region/segment to the other. Regions can be clusters as in [1] or can be in the form of zones as in the case of adhoc routing schemes. Protocols like GPSR and GEAR which try to minimize the energy consumed by considering both energy and distance.

Geographic routing also known as localized algorithms as these algorithms use the location information and transmit the information towards the geographic direction of destination via multiple paths. In Location Aided Routing (LAR), intended to support topology-based reactive routing protocol uses location information to restrict flooding to request zone from where nodes are selected to find the routes between source and destination. ILAR (Improved Location Aided Routing) is another location based routing algorithm, selects the closet neighbor to the base line as an intermediate node.

In [11] authors suggested a load balancing factor that will help in routing with in a WSN but the load balancing factor $(\Theta)$ depends upon the energy of the sensor node. Here the energy is not a static one; hence the proposed scheme yields an average result.

\section{PROPOSED WORK}

The proposed scheme works over a WSN with an assumption that the network spread like a two dimensional planner square grid with equal spacing.

There are some other assumptions are:

- All the nodes are homogeneous in nature.

- The nodes can be traced by its geographical location through available GPS methods.

- The nodes are static in nature at data gathering stage.

- The homogenearity parameters of the nodes are computational power, energy constraint, limited storage and communication range.

The proposed scheme deals with the routing path between a source node to a destination node. There are a number of techniques are available but the proposed scheme highlights over a very efficient geometric technique to find the path with in a very short time.

Here each of the node with in the square grid has eight $(\mathbf{8})$ neighbors as related in Cartesian co-ordinate system(x,y) as $\mathrm{N} 1(\mathrm{x}, \mathrm{y}+1), \mathrm{N} 2(\mathrm{x}, \mathrm{y}-1), \mathrm{N} 3(\mathrm{x}+1, \mathrm{y}), \mathrm{N} 4(\mathrm{x}-$ $1, y), N 5(x+1, y+1), N 6(x+1, y-1), N 7(x-1, y+1), N 8(x-1, y-1)$.

As the geometry suggests that each point $(\mathrm{x}, \mathrm{y})$ belongs to a particular Quadrant depending upon its (x, y) value with respect to the Origin. The proposed scheme first finds the Quadrant of Source(S) and destination(D) then the next node from S's neighbors is selected which belongs to the quadrant of D.Here the whole WSN is sub-divided into four regions based on some point $(0,0)$ as origin. Then the origin is shifted into the source (S) node and all the neighbors (N8) and the destination node (D) s' co-ordinates are adjusted as per geometric formula as mentioned in the figure 2 .

Imagine $\mathrm{O}(0,0)$ is the origin and $\mathrm{S}(\alpha, \beta)$ is the source and $\mathrm{D}(\mathrm{x}, \mathrm{y})$ is the destination ; now if the origin is being shifted into $\mathrm{S}(\alpha, \beta)$ the co-ordinate of $\mathrm{D}(\mathrm{x}, \mathrm{y})$ becomes $\mathrm{D}\left(\mathrm{x}^{\prime}, \mathrm{y}^{\prime}\right)$ as per following :

\section{$X^{\prime}=X-\alpha \quad$ and $\quad Y^{\prime}=Y-\beta$}

The quadrants are set in such a way that the Source and Destination will be in same quadrant.

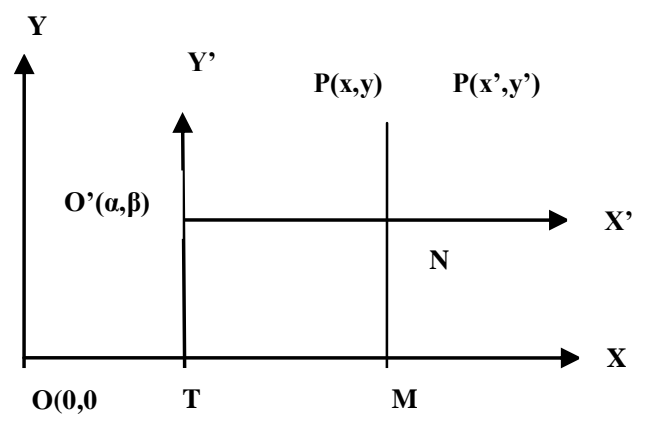

Fig.2. Geometric Origin \& its Shifting

Similarly all the co-ordinates of the neighbors (N8) of source (S) are adjusted with the new origin. Now the actual process starts by filtering the $\mathrm{N} 8$ based on their quadrant location. The selected nodes from N8 are those whose quadrant position is similar with $\mathrm{D}$. Then the perpendicular distance is calculated from that nodes to D and the smallest is selected to become the next hop towards D. Say that node is $N_{x}$; now the $N_{x}$ becomes the next Source $S_{x}$ and the process goes recursively until the $S_{x}$ becomes $D$ (destination).

\section{Flow Chart:}

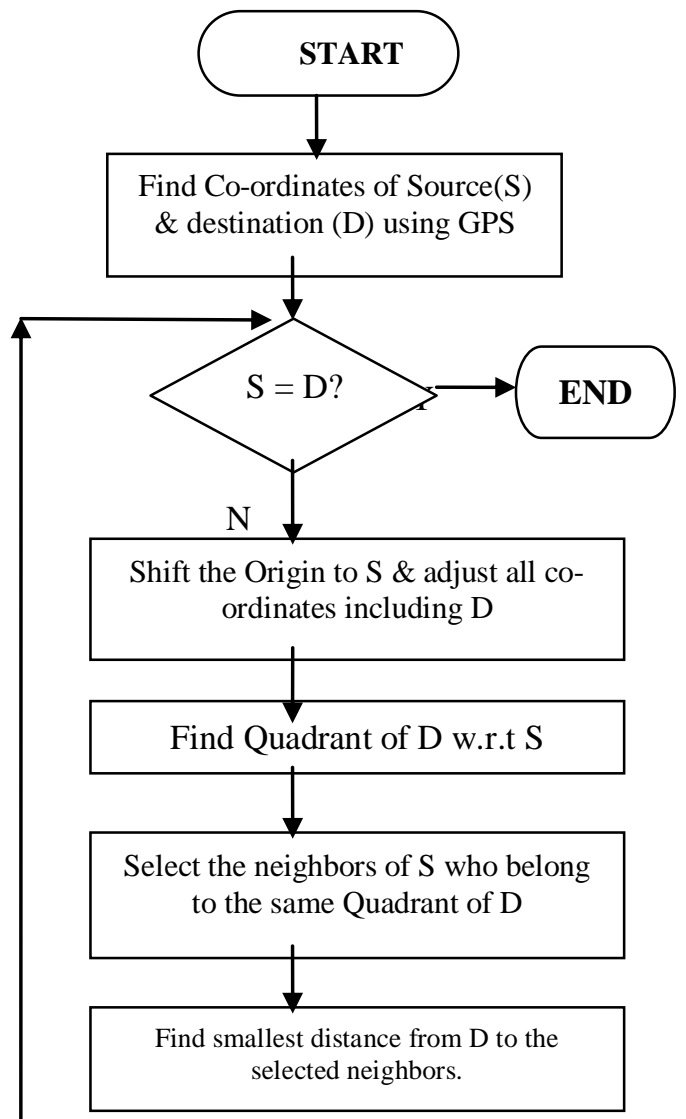




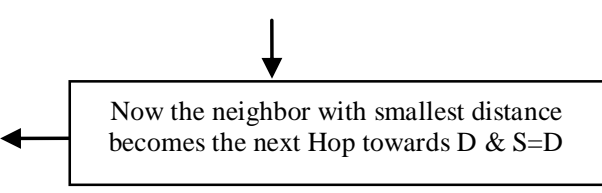

Fig.3.

Flow chart of the proposed Scheme

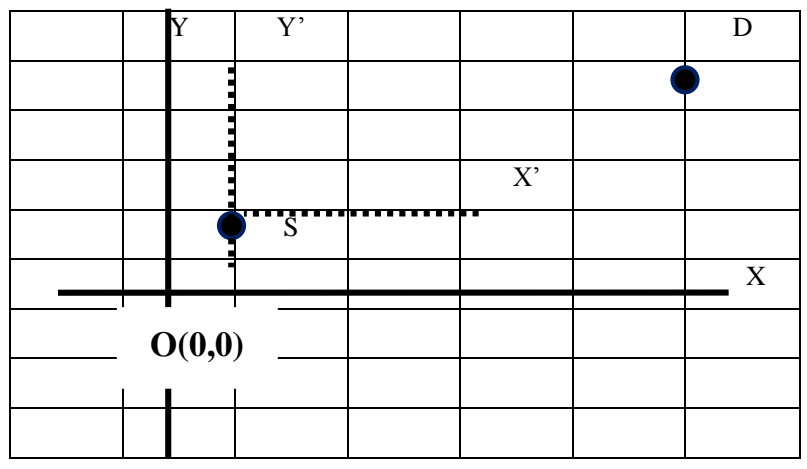

Fig.4. Grid structure with Source \& Destination w.r.t Origin.

Here in the square grid initially the origin is $\mathrm{O}$ the it is shifted at $\mathrm{S}$. Now the adjustment of co-ordinates are taken place with respect to the new origin $\mathrm{S}$.

\section{Example :}

Let origin $\mathrm{O}(0,0)$, Source $\mathrm{S}(5,5)$ and destination $\mathrm{D}(12,9)$. Now the process starts by shifting the origin into $S(5,5)$ hence all the neighbors of S (N8) and Destination D $(12,9)$ will adjust their co-ordinates based on the newer origin. Now D becomes $(12-5,9-5)=(7,4)$ as adjusted co-ordinate. As D is in the first quadrant then the neighbors (from N8) only selected to become a candidate for next hop towards the destination who belongs to the same quadrant with $\mathrm{D}$ (here that is first quadrant).

\section{Simulation ResUlt}

Complexity: As compared to other methods here the complexity of the routing process is reduced a lot as the selection of next hop is done based on a filtering mechanism with the quadrant position of destination node. As there are four quadrants and as per proposed mechanism the selected quadrant is one the mathematically the complexity is reduced up to $25 \%$ of the total efforts.

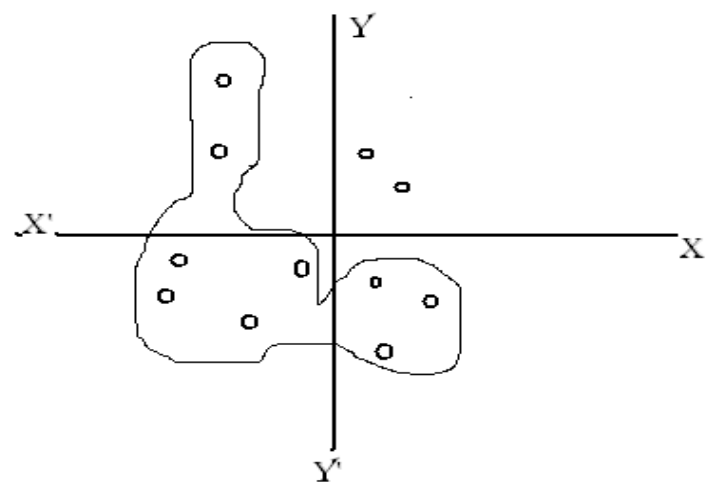

The marked region is the area where the proposed algorithm does not work as it is not associated with the quadrant of $\mathrm{D}$; hence only the unmarked region will take part in the process to become the next hop.

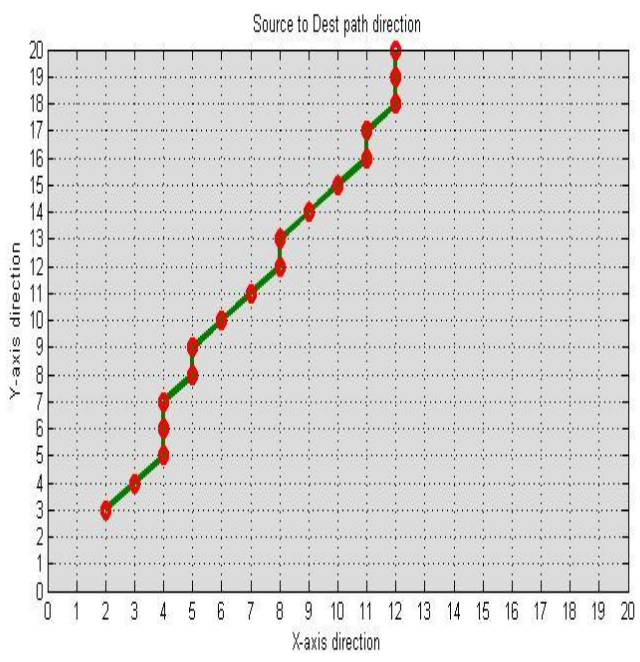

Fig.6.

Output of Simulation

\section{ACKNOWLEDGMENT}

"Quadrant Based WSN Routing Technique By Shifting Of Origin" is an efficient scheme by which we can reduce the effort upto $75 \%$ of the total effort by separating the whole WSN with respect to origin and its 4 quadrants. The proposed scheme will reduce the effort as well as help to preserve battery power of the sensor node.

\section{FUTURE WORK}

The proposed scheme can be extended in the context of energy or power of the sensor node. In [11] the author explained about a threshold value that can be extended in the proposed scheme to efficiently utilize the routing technique in WSN.

\section{References}

[1] Al-Karaki, J., Kamal, A.: Routing techniques in wireless sensor networks: a survey. IEEE Wireless Communications 11(6), 6-28 (2004)

[2] Blaze, M., Feigenbaum, J., Lacy, J.: Decentralized trust management. In: Proceedings of 1996 IEEE Symposium on Security and Privacy, pp. 164-173 (1996)

[3] Boukerche, A., El-Khatib, K., Xu, L., Korba, L.: A novel solution for achieving anonymity in wireless ad hoc networks. In: Proceedings of the 1st ACM international workshop on Performance 3evaluation of wireless ad hoc, sensor, and ubiquitous networks, pp. 30-38 (2004)

[4] Analysis and Improvement of the Dynamic Load Balancing of Overlaybased WSN. By Hang Qin and Zhongbo Wu.

[5] Routing Protocols in Wireless Sensor Networks. By Luis Javier García Villalba , Ana Lucila Sandoval Orozco, Alicia Triviño Cabrera and Cláudia Jacy Barenco Abbas. Sensors 2009, 9, 8399-8421; doi:10.3390/s91108399.

[6] Akkaya, K.; Younis, M. A Survey on Routing Protocols for Wireless Sensor Networks. Ad Hoc Netw. 2005, 3, 325-349.

[7] Tilak, S.; Abu-Ghazaleh, N.B.; Heinzelman, W. A Taxonomy of Wireless Micro-Sensor Network Models. Mob. Comput. Commun. Rev. 2002, 6, 28-36.

Fig.5. Proposed Schemes efficiency 
[8] Seada, K.; Helmy, A. Geographic Protocols in Sensor Networks; Technical Report 04-837; Computer Science Department, University of Southern California: San Diego, CA, USA, 2008.

[9] Lindsey, S.; Raghavendra, C.S. PEGASIS: Power-Efficient Gathering in Sensor Information Systems. In Proceedings of the Aerospace Conference, Big Sky, MT, March, 2002; pp. 1125-1130.

[10] Barenco Abbas, C.J.; González, R.; Cárdenas, N.; García Villalba, L.J. A Proposal of a Wireless Sensor Network Routing Protocol. Telecommun. Syst. 2008, 38, 61-68.

[11] Threshold Based Load Balancing Protocol

for Energy Efficient Routing in WSN. By Sohail Jabbar, Ayesha Ejaz Butt, Najm us Sahar, Abid Ali Minhas . ISBN 978-89-5519-155-4 PP. 196 Feb. 13 16, 2011 ICACT2011.

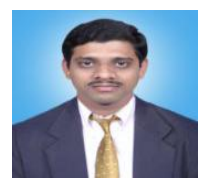

AUTHORS PROFILE

Mr. Nandan Banerji,Pursuing M.Tech(CSE) in the Department of Computer Science and Engineering, University of Kalyani, Kalyani,Nadia, West Bengal, INDIA. His research interest includes wireless and mobile system and WSN

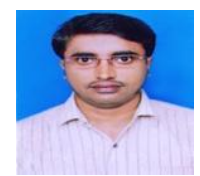

Uttam Kumar kundu Completed his M.Tech in ECE from WBUT in 2010 and B-Tech in E.I.E from JIS College of engineering and technology under West Bengal University of Technology in 2006. His research communication.

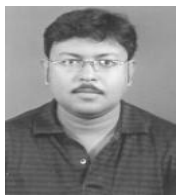

Pulak Mazumder received his M.Tech in Electronics \& Telecommunication Engineering from WBUT, Kolkata in 2010, and his B.Tech(AMIETE) in Electronics \& Telecommunication Engineering from IETE, New Delhi in 2006. At present, he is a Asst. Professor in the Department of Electronics and Tele-Communication Engineering, Regent Education \& Research Foundation, Barracpore. He was earlier a lecturer in ECE dept. at Durgapur Institute of Advanced Technology, Durgapur and Calcutta Institue of Engineering and Mangement, Tollygung, Kolkata. He served Industry more than eight years as IT Network Infrastructure Management. His research interest includes wireless and mobile

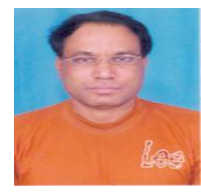
communication systems

Mr Debabrata Sarddar, Assistant Professor in the Department of Computer Science and Engineering, University of Kalyani, Kalyani,Nadia, West Bengal, INDIA. He has done $\mathrm{PhD}$ at Jadavpur University. He completed his M.Tech in Computer Science \& Engineering from DAVV, Indore in 2006, and his B.E in Computer Science \& Engineering from NIT, Durgapur in 2001. He has published more than 75 research papers in different journals and conferences. His research interest includes wireless and mobile system and WSN. 